\title{
Social Media Crisis Communication Model for Building Public Resilience: A Preliminary Study
}

Umar Ali Bukar 1[https://orcid.org/0000-0002-3983-6919], Marzanah A. Jabar 1[https://orcid.org/0000-0002-3619-5028],

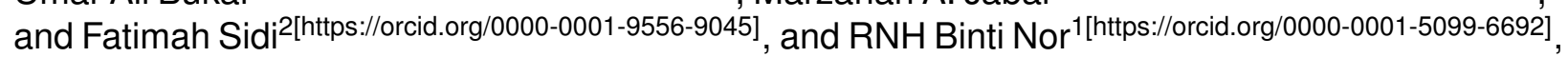
and Salfarina Abdullah ${ }^{1}$ [https://orcid.org/0000-0002-8522-9175]

\footnotetext{
${ }^{1}$ Department of Software Engineering and Information System, Faculty of Computer Science and Information Technology, Universiti Putra Malaysia (UPM), Serdang 43400, Malaysia

${ }^{2}$ Department of Computer Science, Faculty of Computer Science and Information Technology, Universiti Putra
} Malaysia (UPM), Serdang 43400, Malaysia

\begin{abstract}
There is an ongoing discussion about the effectiveness of social media usage on the ability of people to recover from the crisis. However, the existing social media crisis communication models could not address the dynamic feature of social media users and the crisis, respectively. Therefore, the objective of this study is to conduct a preliminary investigation of the social media crisis communication model for building public resilience. Thus, 34 items were generated from the literature concerning the crisis, crisis response, social interaction, and resilience. The items were validated by three experts via content validity index and modified kappa statistics. After passing the validation test, the instruments were pre-tested by 32 participants. The reliability of the items was analyzed using Cronbach's alpha. Also, the model fits and mediation were examined by the regression model, and the hypotheses were independently assessed in process macro models. Based on the result obtained, each of the constructs satisfied the internal consistency requirement; crisis $(0.743)$, crisis response $(0.724)$, social media interaction (0.716), and resilience (0.827). Furthermore, the result also indicates that the regression model is a good fit for the $d$ ata. The independent variables statistically significantly predict the dependent variable, $p<0.05$. Also, the result of the process macro models indicates that all the hypotheses are independently supported.
\end{abstract}

Keywords: Crisis communication, Social media, Resilience, Social interaction, Crisis response

\section{Introduction}

The widespread adoption of technology has enabled crisis response and humanitarian development to be considered the future of human progress and well-being (1). Crisis management is driven by advances in computing, communications, storage, processing, and analysis. Technology-driven emergency management is continuously evolving as a new research field. Each step to improve methods or tools can make a significant contribution to saving human lives and resources. Emergency management, disaster management, and crisis management are often used interchangeably (2). Their role is to coordinate efficient actions related to information dissemination, security, supply, lodging in a highly dynamic and uncertain environment (3). The occurrence of a crisis, in particular, a disaster, is hard to predict, but its effects can be minimized through enabling technologies (4).

Crises come with a rapid increase in communication and a decrease in physical interactions. Coronavirus (Covid-19) is an example of a crisis that has become a threat to interaction 
as everyone tries to have little interaction as possible (5) (World Health Organisation [WHO]. Information about the virus is spread on social networking sites which assists situational awareness, stakeholders interactions, and crisis responses (6). Social media enabled groups and individuals to collaborate and engage in crisis communication which also enabled digital convergence of people, information, and resources during crises (7). As a result, the people are far from being passive receivers; they actively seek out crisis information and exchange views with others (8). Hence, the dependence of formal and informal stakeholders (management and public) response is an established requisite for effective crisis communication and management

(9; 10). Social media hypothetically intensifies the influence of the public's responses (10).

Therefore, this study aims to investigates the impact of the crisis, crisis response, and social media interaction on community resilience as part of an ongoing study on modeling social media crisis communication for building resilience. Therefore, the section "related work" discusses a few works that study the interaction among various stakeholders involved in crisis response on social media, the existing theories, and the conceptual model. The section "methodology" discusses the data and measurement method. The section "result and findings" presents the result of the content validity index, modified kappa, Cronbach's alpha, and regression model. The section "discussion" presents our argument, and finally, the section "conclusion" presents our concluding remarks and future research.

\section{Related Work}

The most dominant and commonly used theory to examine crisis management and communication strategies is the situational crisis communication theory (SCCT) (11; 12). The socialmediated crisis communication (SMCC) model classified social media public as influencers (content creators), followers, and inactive users (13; 14). The integrated crisis mapping (ICM) conceptualizes stakeholders' emotions (15). Reference (16) integrated the SCCT, SMCC, and ICM to link stakeholders' emotions and response strategies. Furthermore, (17) proposed the STREMII model, particularly for social media crisis management, is dynamic and cyclic, but the model is limited to response and recovery (18). Also, a new integrated crisis mapping approach based on ICM provides a general approach and directions for building a crisis communication model and a direct way of handling crisis response for effective reactions of public emotions (19). Moreover, the social media disaster resilience (SMDR) model has demonstrated how social media usage improves community resilience during and after crisis (20). Additionally, reference (21) introduced an interactive crisis communication model (ICCM) based on SCCT, SMCC, and traditional crisis communication strategies (CCS). ICCM is the first model that provides an integrated strategy toolkit that synthesizes SCCT and CCS crisis response strategies. The major weakness of ICCM is its failure to investigate the impact of interaction among stakeholders. The theoretical models and hypotheses are discuss as follows;

\section{Situational Crisis Communication Theory (SCCT)}

Situational crisis communication theory (SCCT) was developed in 1995, refined, and renamed in 2002 (11). The SCCT was motivated by the absence of a model to connects crisis to crisis response strategies (what crisis communicators say and do during a crisis) and crisis situations. The SCCT connects crisis and crisis response strategies and crisis types through the lens of attribution theory. Through crisis response, individuals seek to comprehend why a crisis event occurred. The work by (11) highlighted the importance of attributions, stating that they influence how people feel and react to an event. According to SCCT, crises are adverse events that cause people to judge the crisis management authorities, and a timely response safeguards the crisis management reputations (22). The digital environment, mainly social media platforms, enables individuals to challenge crisis management (11) due to public engagement in crisis response via social media, which also aids in the recovery process. On social media, the crisis has an impact on how crisis response is formed and led. Thus, crisis response and social media 
interaction are used to address the situation when the crisis occurs. This demonstrated the effectiveness of crisis response and social media interaction in assisting people recovering from a crisis. Thus, the research framework (Figure 2) indicates that crisis significantly affects resilience, social media interaction, and crisis response, as the following hypothesis suggests.

- Hypothesis $1(\mathrm{H} 1)$ : Crisis has a significant impact on resilience.

- Hypothesis $2(\mathrm{H} 2)$ : Crisis has a significant impact on the crisis response.

- Hypothesis $3(\mathrm{H} 3)$ : Crisis has a significant impact on social media interaction.

Additionally, crisis response refers to the response of stakeholders (both public and management) to a crisis. This is discussed in the social-mediated crisis communication model (SMCC), which focuses on the types of public, the sources of information, and the information format. The crisis response enables stakeholders to create content that expresses their views on/about the crisis or the entity managing it. Understanding the full range of public emotions enhances the effectiveness of crisis response strategies (14; 16; 17), all of which have an effect on the public's ability to recover. The crisis management literature frequently refers to three stages of crisis management as para-crisis, crisis, and post-crisis $(14 ; 18 ; 21 ; 23)$. As is the case with crisis responses, the nature of the para-crisis is considered to determine the paracrisis response that will most effectively mitigate the crisis risk (11). Thus, crisis response has an effect on resilience and social media interaction. Hence, the crisis-resilience relationship is mediated by crisis response and social media interaction. Therefore, this study implies that crisis response acts as a mediator between crisis and social interaction, as well as between crisis and resilience, as described in the hypothesis below.

- Hypothesis $4(\mathrm{H} 4)$ : Crisis response has a significant impact on resilience.

- Hypothesis 5 (H5): Crisis response has a significant impact on social media interaction.

- Hypothesis $7(\mathrm{H} 7)$ : The relationship between crisis and social media interaction is mediated by crisis response.

- Hypothesis $8(\mathrm{H} 8)$ : The relationship between crisis and resilience is mediated by crisis response.

\section{Interactive Crisis Communication Model (ICCM)}

The interactive crisis communication model (ICCM), introduced by (21), is relatively new in the crisis communication literature, which is base on SCCT, SMCC, and traditional crisis communication strategies (CCS). The ICCM is built for social media, demonstrating and representing the total interaction of stakeholders in a digital environment. Since everyone participates in crisis response, the model reaffirms the SMCC classification of public engagement and interactions. The ICCM demonstrates the importance of social interaction by demonstrating its capacity to provide one of the four gratifications identified in the uses and gratification theory (UGT) (24; 25). The entire ICCM is referred to as an interactive model, as it illustrates the fundamental elements of crisis management's interaction with the public. Similarly, the interaction is a fundamental component of the STREMII model (17). The ICCM demonstrates why social interaction is critical in social media crisis response. According to the ICCM model, since social media is an object or environment that enables groups and individuals to collaborate, the content can take the form of text, visual, audio, or a combination of these, which referred to as the interaction's content $(14 ; 17 ; 20 ; 21)$. The contents are from two sources: crisis management and public response. The purpose of this study is to examine the effect of social interaction on resilience. Thus, this study implies that there is a relationship between social media interaction and resilience. As demonstrated in the hypothesis below, social media interaction serves as a mediator between crisis and resilience and crisis response and resilience.

- Hypothesis $6(\mathrm{H} 6)$ : Social media interaction has a significant impact on resilience.

- Hypothesis $9(\mathrm{H} 9)$ : The relationship between crisis and resilience is mediated by social media interaction. 
- Hypothesis $10(\mathrm{H} 10)$ : The relationship between crisis response and resilience is mediated by social media interaction.

\section{Social Media Disaster Resilience (SMDR) Model}

The social-mediated disaster resilience (SMDR) model was introduced by (20), who demonstrated how social media usage is integrated into resilience building and discusses its potential for increasing hotel resilience. The study links resilience and disaster management literature using the revised $3 \mathrm{Rs}$ (robustness, rapidity, and redundancy) resilience model. Then, discussed social media as a robust technology to be used in crisis(13), to increase the speed of communication and information distribution (rapidity) $(26 ; 27)$, and to redistribute the targeted information to a larger crowd via crowdsourcing (redundancy). In general, there is enough literature on how social media detects and document disasters (28), send and receive assistance (29), spread warnings $(30)$, and solicit donations and volunteerism $(29 ; 30)$. Thus, the robustness of social media has enabled the public to participate in crisis communication discussions, establishing them as vital resources (31). Additionally, the information can be quickly and widely distributed via social media crisis response and social interaction. While resistance is the first ideal outcome following a crisis, robustness, rapidity, and redundancy (3Rs) are critical for increasing resilience to the adverse effects of a crisis or disaster (20). Hence, it is essential to investigate the impact of crisis management efforts since their role is to strengthen relationships and improves the community's resilience $(14 ; 20)$. Accordingly, crisis response and social media interaction mediates the crisis-resilience relationship as the following hypothesis suggest.

- Hypothesis $11(\mathrm{H} 11)$ : The relationship between crisis and resilience is mediated by crisis response and social media interaction.

\section{Conceptual Model}

According to reference (12), in the effort to represent the nature of the interactions between various stakeholders and how this study concept is different from existing studies of crisis communication. The study reiterates this concept and introduces the ICCM model representing the interaction between crisis management and the public on the social media environment (21) as reported in the STREMII model (17). Then adopted the resilience concept from the SMDR model that shows how social media usage improves community resilience (20).

First, since the crisis is the trigger that allows crisis response (32) to take place on social media, the nature of the crisis and crisis response are factors influencing stakeholder's formation on social media. These reflect the unmet challenge of integrating qualitative and quantitative modeling to understand how interaction, leadership, and social structure are represented in electronic trace data generated due to crisis and stakeholder's crisis responses (33). Second, the online contexts in which stakeholders interact as sociotechnical interaction places as represented in ICCM (21). This indicates where peoples interact as groups for a specific purpose and mediate consistent and meaningful aspects of their activity through technology that generates electronic trace data. The entire ICCM represents social interaction between stakeholders involves in crisis responses. Third, the SMDR proved the use of social media for building resilience but does not investigate the impact of social media usage or interaction through quantitative means. Therefore, the conceptual model (presented in Figure 2 along with the result) intends to show how social media interactions can improve community resilience since crisis management actions aim to improve the relationship and increase community resilience. The interactions between stakeholders (management and public) due to crisis can show the intensity of crisis response on social media(14; 20). 


\section{Methods}

\section{Data and Measures}

The questionnaire's items are derived from existing literature $(20 ; 25)$. The expert evaluation was developed based on these items, including discussing each construct, item, and how the existing theories were linked to form the relationship between the variables. Then, the evaluation document was sent to the expert for content and face validity. There are several recommendations for performing content validity $(25 ; 34 ; 35)$. In this study, the validation uses three experts in crisis informatics and crisis communication, which have over 50years of combined working experience.

The expert evaluation form consist of 34 items distributed among the four construct; crisis = 6 , crisis response $=6$, social media interaction $=7$, and resilience $=15$. For content validation, several studies are encouraging using a 4-point Likert scale to avoid aggregations problems (34; 35). Therefore, each item was measured using 4-point Likert scales ranging from 1 (not relevant) to 4 (highly relevant) for the expert evaluation phase to examine if the items are appropriate to measure what it intends to measure. Notably, the expert encouraged the use of a 5-point Likert for further study. After passing the early validation phase, the questionnaire was designed via an online platform (google form) on 5-point Likert scales with a range from 1 (strongly agree) to 5 (strongly disagree). The answers were received within one week, with 32 completed responses. Study participants must have experienced Covid-19 lockdown and have observed social distancing rules to avoid the crowded area, self-isolate, or quarantine. The respondents were mainly males (approximately $85 \%$ ) and are highly educated; $94 \%$ held a postgraduate or master's degree. In terms of age, most respondents were in the age range of 35-44 $(51.5 \%)$ or 25-34 (33.3\%). Approximately $12.1 \%$ were between 45 and above years old, and about $3.1 \%$ were between 18 - 24 years old. The sample was considered appropriate for the preliminary test (pilot study) of the study.

\section{Method of Analysis}

The analysis methods are considered threefold; the content validity method, the reliability, and the assessment model fit, and the mediation fit model. Therefore, the content validity index (CVI) is considered the most widely used method to validate research items (34; 36). The CVI expressed and classified the proportion of agreement into either 0 or 1 . Although the significant weakness of CVI is its failure to adjust for chance agreement and the researchers suggest the modified kappa statistics to address chance agreement $(34 ; 35 ; 37)$. Studies encouraging the use of a 4-point Likert scale for CVI specified that the rating of 1 and 2 are considered content invalid while those from 3 and 4 to be content valid $(34 ; 35 ; 36 ; 37)$. Therefore, the 4-point rating is collapsed into dichotomous categories of responses. Any response that falls between 1 and 2 is assigned 0 , and those that fall in 3 and 4 are assigned 1 . Secondly, the instrument's reliability and the relationship between the independent variables (IVs) and the dependent variable (DV) were measured using Cronbach's alpha and regression model. Thirdly, the hypotheses were independently assessed in PROCESS macro models (38), and the indirect effect was tested using a percentile bootstrap estimation approach with 5000 samples (39) on SPSS version 24.

\section{Result and Findings}

\section{Content Validation}

Several studies have provided information on how to sufficiently measure the value that indicates a high level of agreement among the raters $(34 ; 35 ; 37 ; 36)$. (34) emphasized that an average value of $70 \%$ is necessary for agreement, $80 \%$ for adequate agreement, and $90 \%$ for a good agreement. In comparison, other researchers recommend values between 70 and 79 percent to be considered for revision while less than $70 \%$ to be eliminated (35; 37 ). Also, few 
suggestions are also employed to measure the strength of agreement for kappa which classified as less than 0.40 as poor, $0.40-0.59$ as fair, $0.60-0.74$ as good, and $0.75-1.00$ as excellent (34; 37). The result of CVI and modified kappa statistics are excellent for this study. All the items indicate an excellent level of agreement among the expert. The value for I-CVI and Modified kappa for each item is 1 .

\section{Reliability and Consistency of the Instrument}

The Cronbach's alpha assessed scale reliability, and the alpha for each construct is presented in Table 1.

Table 1. Crombah Alpha Reliability Parameters of the Constructs

\begin{tabular}{lrr}
\hline Constructs & No of items & Crombach Alpha \\
\hline Crisis & 6 & 0.743 \\
Crisis response & $6-1(5)$ & 0.724 \\
Social media interaction & 7 & 0.716 \\
Resilience & 15 & 0.827 \\
\hline
\end{tabular}

\section{Model Analysis}

Multiple regression was applied to predict resilience from the crisis, crisis response, and social media interaction. These variables statistically significantly predicted resilience, $F(3,95)=$ $15.141, \mathrm{p}<0.05, R^{2}=0.619$. The standard error of the estimate determines how the model fits the data. A rule of thumb R-value is considered a measure of predicting the DV; in this case, resilience. A value of 0.787 indicates a good level of model fits. Also, the $R$ Square value, which is 0.619 , shows that the IVs explain $61.9 \%$ of the variability of the DV.

In Table 2, the overall model is tested to determine if the regression model is a good fit for the data. The result of the analysis of variance (ANOVA) indicates that the IVs statistically significantly predicts the $D V, p<0.05$. The results indicated that the overall model is a good fit for the data.

Table 2. Analysis of Variance (ANOVA)

\begin{tabular}{lrrrrr}
\hline Model 1 & Sum of Squares & Df & Mean Square & F & Sig. \\
\hline Regression & 3.056 & 3 & 1.019 & 15.141 & $.000^{b}$ \\
Residual & 1.884 & 28 & 0.067 & & \\
s Total & 4.94 & 31 & & & \\
\hline
\end{tabular}

a. Dependent Variable: Resilience

b. Predictors: (Constant), Interaction, CrisisResponse, Crisis

Figure 1 presents the results for multivariate normality and linear relationship between the IVs and the DV. The results in Figure 1 (left) indicate that the multivariate normality is present in the data, normally distributed. Secondly, in Figure 1 (right), the results suggested an excellent linear relationship between the IVs and the DV.

\section{Path and Mediation Analysis}

Results are obtained concerning the relationship between the IVs and the DV and whether social media interaction acted as a mediator between crisis and resilience and between crisis response and resilience. Additionally, results are obtained for whether crisis response acted as a mediator between crisis and resilience. Figure 2 presents the impact of the crisis, crisis response, and social media interaction on resilience.

The results indicated that crisis is a significant predictor of resilience, $\beta=0.3155$, SE $=$ $0.1318, \mathrm{p}<0.05$, crisis response, $\beta=0.5480$, $\mathrm{SE}=0.1795, \mathrm{p}<0.05$, and social media interaction, $\beta=0.6636$, SE $=0.1413, p<0.05$, indicating support for $\mathrm{H} 1, \mathrm{H} 2$, and $\mathrm{H} 3$. Similarly, 

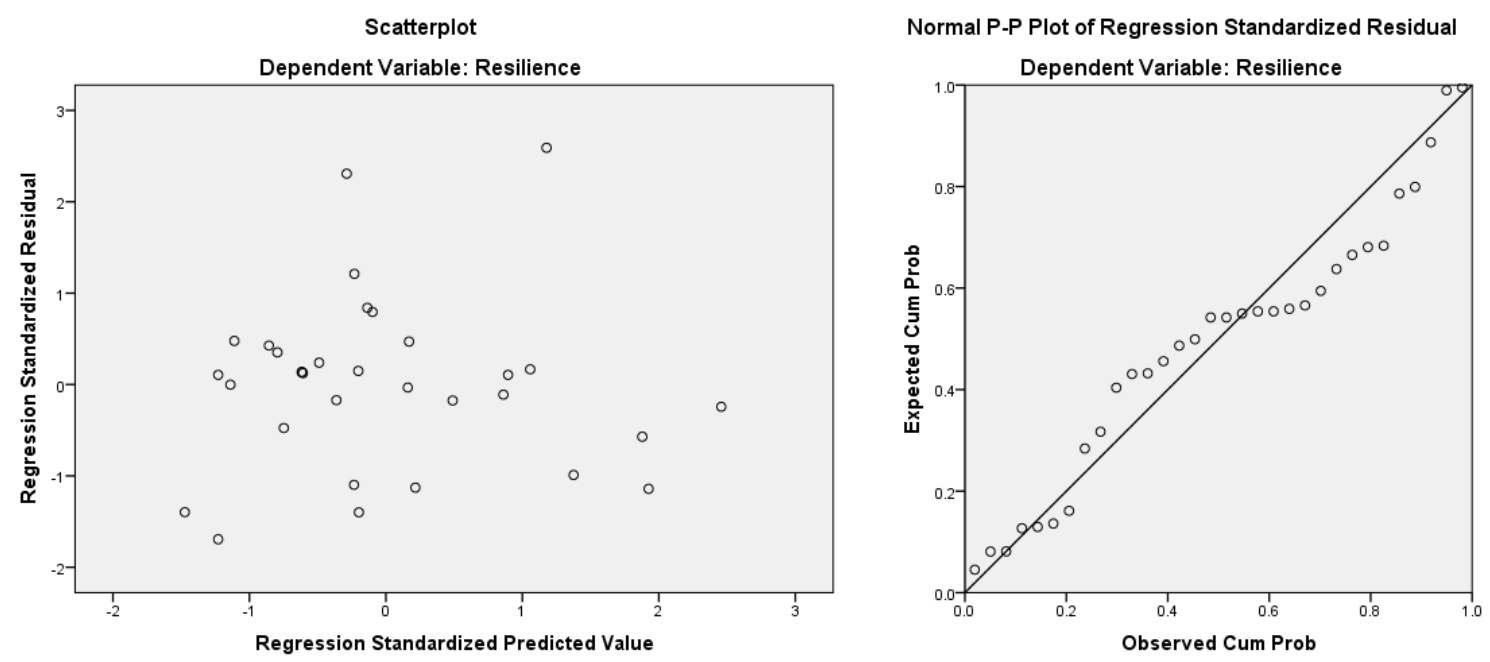

Figure 1. Result for Multivariate Normality of IV and DV (left) and Relationship Between IV and DV (right)

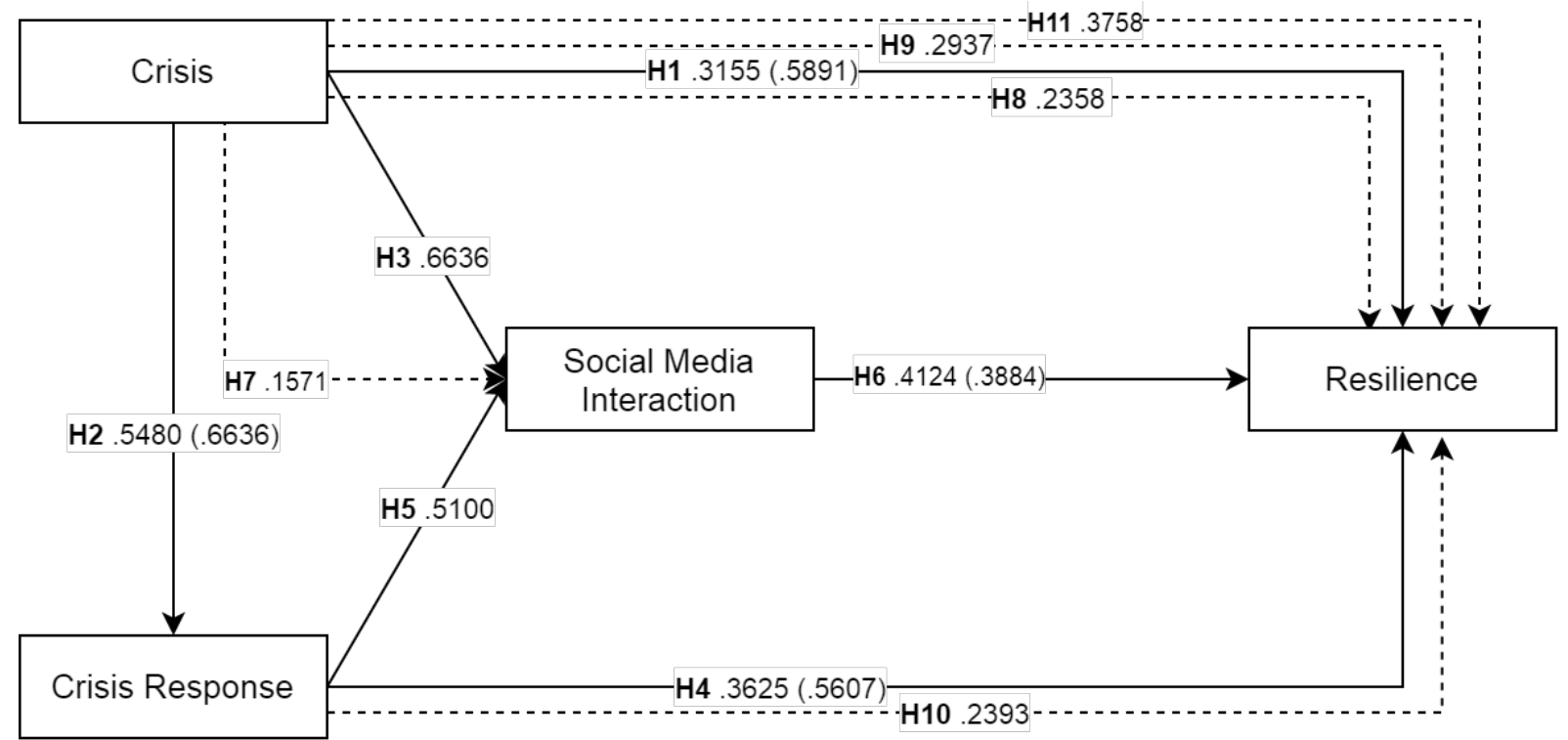

Figure 2. A Model for Social Media Crisis Communication for Resilience

the results indicated that crisis response is a significant predictor of resilience, $\beta=0.3625, \mathrm{SE}$ $=0.1112, \mathrm{p}<0.05$ and social media interaction, $\beta=0.5100, \mathrm{SE}=0.1367, \mathrm{p}<0.05$, indicating support for $\mathrm{H} 4$ and $\mathrm{H} 5$. Also, social media interaction is a significant predictor of resilience, $\beta=$ 0.4124 , SE $=0.1553, \mathrm{p}<0.05$, indicating support for $\mathrm{H} 6$.

The results of mediation was observed. The results for the indirect effect of social media interaction on crisis-crisis response relationship indicated that the indirect coefficient was significant, $\beta=0.1571$, $\mathrm{SE}=0.0969,95 \% \mathrm{Cl}=0.0145,0.3898$, indicating support for $\mathrm{H} 7$. The proportion of the total effect of crisis on crisis response is $\beta=0.6636, \mathrm{SE}=0.1413, \mathrm{p}<0.05, R^{2}$ $=0.4237$, is indirectly significant by $42.37 \%$. Moreover, the results for the indirect effect of crisis response on crisis-resilience relationship indicated that the indirect coefficient was significant, $\beta=0.2358, \mathrm{SE}=0.0958,95 \% \mathrm{Cl}=0.0625,0.4465$, indicating support for $\mathrm{H} 8$. The proportion of the total effect of crisis on resilience is $\beta=0.5891$, SE $=0.1318, \mathrm{p}<0.05, R^{2}=0.3998$, is indirectly significant by $39.98 \%$. Moreover, the results for the indirect effect of social media interaction on crisis-resilience relationship indicated that the indirect coefficient was significant, $\beta=0.2937, \mathrm{SE}=0.1598,95 \% \mathrm{Cl}=0.0834,0.7250$, indicating support for $\mathrm{H} 9$. The proportion 
of the total effect of crisis on resilience is $\beta=0.5891$, SE $=0.1318, \mathrm{p}<0.05, R^{2}=0.3998$, is indirectly significant by $39.98 \%$. Additionally, the results for the indirect effect of social media interaction on crisis response-resilience relationship indicated that the indirect coefficient was significant, $\beta=0.2393, \mathrm{SE}=0.1152,95 \% \mathrm{Cl}=0.0576,0.5003$, indicating support $\mathrm{H} 10$. The proportion of the total effect of crisis response on resilience is $\beta=0.5607$, SE $=0.1112, p<$ $0.05, R^{2}=0.4587$, is indirectly significant by $45.87 \%$. Finally, the results for the indirect effect of social media interaction and crisis response on crisis-resilience relationship indicated that the indirect coefficient was significant, $\beta=0.3758$, SE $=0.1385,95 \% \mathrm{Cl}=0.1499,0.7269$, indicating support for $\mathrm{H} 11$. The proportion of the total effect of crisis on resilience is $\beta=0.5891$, $\mathrm{SE}=0.1318, \mathrm{p}<0.05, R^{2}=0.3993$, is indirectly significant by $39.93 \%$.

\section{Result Summary}

The expert validation results show that all items indicate excellent agreement both from CVI and modified kappa statistics. The scale reliability and internal consistency were measures using Cronbach's alpha, and the results observed that one of the items has less scored by the participants. This item was not considered in the linear regression model and process macro model, which is left for further study with a large sample size. Furthermore, the mediation analysis based on the regression coefficients supported all the hypotheses, see Table 3.

Table 3. Hypothesis Assessment of the Direct and Indirect Effects

\begin{tabular}{lll}
\hline S/N & Hypotheses & Decision \\
\hline H1 & Crisis $->$ Resilience & Supported \\
H2 & Crisis $->$ Crisis response & Supported \\
H3 & Crisis $->$ Social media interaction & Supported \\
H4 & Crisis response $->$ Resilience & Supported \\
H5 & Crisis response $->$ Social media interaction & Supported \\
H6 & Social media interaction $->$ Resilience & Supported \\
H7 & Crisis $->$ Crisis response $->$ Social media interaction & Supported \\
H8 & Crisis $->$ Crisis response $->$ Resilience & Supported \\
H9 & Crisis $->$ Social media interaction $->$ Resilience & Supported \\
H10 & Crisis response $->$ Social media interaction $->$ Resilience & Supported \\
H11 & Crisis $->$ Crisis response $->$ Social media interaction $->$ Resilience & Supported \\
\hline
\end{tabular}

\section{Discussion}

The discussion about the potentials of social media to improve stakeholders' relationships and increase community resilience called for empirical investigation of this research. As the central aspect of this work, the study conducted a preliminary investigation for social media crisis communication and resilience that offer the basics to determine effective social media-based crisis communication and management. Thus, a particular discussion is about the results and findings achieved from the evaluation of the pilot study. According to the findings, all the research hypotheses were supported. The findings indicated that the public ability to recover from a crisis could be improved through social media-based crisis communication by meaningful engagement in crisis response and social interaction on social media.

Firstly, the findings of this study support existing works $(21 ; 20 ; 17)$, whose conceptualizations of a social media-based crisis communication model emphasized the critical nature of crisis response and social interaction via social media use. These studies laid the groundwork for the model proposed in this paper. However, a few studies have examined the various stakeholders involved in crisis communication on social media $(40 ; 41$; 42$)$ with focused on actors in the issue arena, knowledge about stakeholders relationships, and content of crisis communication. Reasonably, this study may serve as evidence that the goal of crisis informatics is to comprehend the interaction of stakeholders involved in crisis communication (43). Thus, this 
study contributes to the advancement of social media use for crisis communication.

Secondly, reference (44) stated that the long-term impact of the Covid-19 pandemic is unknown, but it is undoubtedly going to last longer than anticipated. One of the pandemic's most significant and immediate effects is how it has shattered the public relationship. Restriction of movement creates a stressful awareness that one's well-being is dependent on others. The pandemic severely harmed individuals' capacity to have actual close relationships with other people, severely harmed the extreme human need for contact, and discouraging any other physical affection and connection. Whereas the explosion of electronic-mediated interaction in recent decades, most notably via social media, has aided people in recovering from crises, demonstrating how critical in-person interaction via social media is building public resilience during the pandemic.

Thirdly, individuals were unable to participate in social activities during the pandemic period. Thus, this study responds to a call for additional research into the short and long-term consequences of critical life-course changes. The crisis and physical separation have the potential to have both immediate and long-term effects on relationships. Some people are susceptible to changes in their environment, especially children. When isolation began, there was considerable anxiety about the infection entering and spreading through the families, and household members struggled to express affection securely physically. Interestingly, social media activities (crisis response and social interaction) have aided significantly in effectively managing physical distancing in these circumstances. Thus, the proposed model demonstrates the future direction of crisis communication to assist affected citizens during such times.

Additionally, when schools are closed, children and adolescents cannot interact face-toface with their friends. Peer gatherings and fellowships are critical for teenagers' character and identity exploration. Social media enabled friendships to endure and engagement to occur. Similarly, despite the availability of various platforms to mitigate the impact of restricted human movement, there were still concerns about the public's emotions, needs for fulfillment, and relationship survival. As a result, the model validated in this study established the requirements and framework for providing effective crisis communication to address these concerns. Finally, the pandemic displaced many people from their jobs, forcing them to collaborate and maintain associations remotely and distantly. Surprisingly, the relationships between the variables examined in this study may aid crisis management organizations in re-positioning their crisis communication activities more effectively.

\section{Conclusion}

This study reported the preliminary test of the impact of the crisis, crisis response, and social media interaction on resilience building. CVI was used for content validation, while Cronbach alpha and regression analysis was incorporated to check the reliability, model fits, and mediation fits. The findings show that the content validity shows excellent agreement among the raters. The internal consistency of each variable meets the required minimum for acceptance, and the regression model is significant.

This study is not without limitations, and the major one is that the test sample is very homogeneous. The respondents were mainly male and mainly from a particular age group. This makes it hard to generalize the finding to women or other age groups, who are also affected by crises. In the future, we intend to collect data from large sample size to investigates the impact of the crisis, crisis response, and social media interaction. Also since the model suggested some attributes of social media interaction such as time distance, type (mode), content, frequency, duration, and intensity. Further work could collect the content of crisis responses from social networking sites based on these attributes. The content can be analyzed using sentiment analysis to understand the intensity of the interaction among stakeholders. 


\section{Acknowledgement}

The authors would like to express gratitude for the financial support provided under the Fundamental Research Grant Scheme (FRGS) through the Grant Cost Centre under FRGS/1/2019/ICT04/UPM/02/5, Vote Number: 5540287

\section{References}

[1] J. Qadir, A. Ali, R. ur Rasool, A. Zwitter, A. Sathiaseelan, and J. Crowcroft, "Crisis analytics: big data-driven crisis response," Journal of International Humanitarian Action, vol. 1, no. 1, p. 12, 2016.

[2] V. Mijović, N. Tomašević, V. Janev, and S. Vraneš, "Event-driven decision support system for intelligent emergency management in critical infrastructures."

[3] M. De Brito, L. Thévin, C. Garbay, O. Boissier, and J. F. Hübner, "Supporting flexible regulation of crisis management by means of situated artificial institution," Frontiers of Information Technology \& Electronic Engineering, vol. 17, no. 4, pp. 309-324, 2016.

[4] K. Starbird, L. Palen, A. L. Hughes, and S. Vieweg, "Chatter on the red: what hazards threat reveals about the social life of microblogged information," in Proceedings of the 2010 ACM conference on Computer supported cooperative work, 2010, pp. 241-250.

[5] W. H. Organization et al., "Novel coronavirus ( 2019-ncov): situation report, 3," 2020.

[6] T. Onorati, P. Díaz, and B. Carrion, "From social networks to emergency operation centers: A semantic visualization approach," Future Generation Computer Systems, vol. 95, pp. 829-840, 2019.

[7] L. Palen and S. B. Liu, "Citizen communications in crisis: anticipating a future of ictsupported public participation," in Proceedings of the SIGCHI conference on Human factors in computing systems, 2007, pp. 727-736.

[8] Y. Ji and S. Kim, "Communication-mediated psychological mechanisms of chinese publics' post-crisis corporate associations and government associations," Journal of Contingencies and Crisis Management, vol. 27, no. 2, pp. 182-194, 2019.

[9] L. Palen, K. M. Anderson, G. Mark, J. Martin, D. Sicker, M. Palmer, and D. Grunwald, "A vision for technology-mediated support for public participation \& assistance in mass emergencies \& disasters," ACM-BCS Visions of Computer Science 2010, pp. 1-12, 2010.

[10] H. Purohit, A. Hampton, S. Bhatt, V. L. Shalin, A. P. Sheth, and J. M. Flach, "Identifying seekers and suppliers in social media communities to support crisis coordination," Computer Supported Cooperative Work (CSCW), vol. 23, no. 4-6, pp. 513-545, 2014.

[11] W. T. Coombs, "Revising situational crisis communication theory," Social media and crisis communication, vol. 1, pp. 21-37, 2017.

[12] U. A. Bukar, M. A. Jabar, F. Sidi, R. N. H. Nor, S. Abdullah, and M. Othman, "Crisis informatics in the context of social media crisis communication: Theoretical models, taxonomy, and open issues," IEEE Access, vol. 8, pp. 1-1, 2020.

[13] J. Yin, A. Lampert, M. Cameron, B. Robinson, and R. Power, "Using social media to enhance emergency situation awareness," IEEE Annals of the History of Computing, vol. 27, no. 06 , pp. 52-59, 2012. 
[14] B. F. Liu, L. Austin, and Y. Jin, "How publics respond to crisis communication strategies: The interplay of information form and source," Public Relations Review, vol. 37, no. 4, pp. 345-353, 2011.

[15] Y. Jin, A. Pang, and G. T. Cameron, "Toward a publics-driven, emotion-based conceptualization in crisis communication: Unearthing dominant emotions in multi-staged testing of the integrated crisis mapping (icm) model," Journal of Public Relations Research, vol. 24, no. 3, pp. 266-298, 2012.

[16] C. Vignal Lambret and E. Barki, "Social media crisis management: Aligning corporate response strategies with stakeholders' emotions online," Journal of Contingencies and Crisis Management, vol. 26, no. 2, pp. 295-305, 2018.

[17] M. C. Stewart and B. G. Wilson, "The dynamic role of social media during hurricane\# sandy: An introduction of the stremii model to weather the storm of the crisis lifecycle," Computers in Human Behavior, vol. 54, pp. 639-646, 2016.

[18] R. Syed, "Enterprise reputation threats on social media: A case of data breach framing," The Journal of Strategic Information Systems, vol. 28, no. 3, pp. 257-274, 2019.

[19] Y. Jin, J.-S. Lin, B. Gilbreath, and Y.-I. Lee, "Motivations, consumption emotions, and temporal orientations in social media use: A strategic approach to engaging stakeholders across platforms," International Journal of Strategic Communication, vol. 11, no. 2, pp. 115-132, 2017.

[20] C. Möller, J. Wang, and H. T. Nguyen, "\# strongerthanwinston: Tourism and crisis communication through facebook following tropical cyclones in fiji," Tourism Management, vol. 69, pp. 272-284, 2018.

[21] Y. Cheng, "How social media is changing crisis communication strategies: Evidence from the updated literature," Journal of Contingencies and Crisis Management, vol. 26, no. 1, pp. 58-68, 2018.

[22] W. T. Coombs, "Attribution theory as a guide for post-crisis communication research," Public Relations Review, vol. 33, no. 2, pp. 135-139, 2007.

[23] W. T. Coombs and J. S. Holladay, "The paracrisis: The challenges created by publicly managing crisis prevention," Public Relations Review, vol. 38, no. 3, pp. 408-415, 2012.

[24] A. Whiting and D. Williams, "Why people use social media: a uses and gratifications approach," Qualitative Market Research: An International Journal, 2013.

[25] Y. Li, S. Yang, S. Zhang, and W. Zhang, "Mobile social media use intention in emergencies among gen y in china: An integrative framework of gratifications, task-technology fit, and media dependency," Telematics and Informatics, vol. 42, p. 101244, 2019.

[26] C. Ehnis and D. Bunker, "Social media in disaster response: Queensland police servicepublic engagement during the 2011 floods," 2012.

[27] M. Irons, "'we can help": an australian case study of post-disaster online convergence and community resilience," Ph.D. dissertation, University of Tasmania, 2015.

[28] C. Reuter and M.-A. Kaufhold, "Fifteen years of social media in emergencies: a retrospective review and future directions for crisis informatics," Journal of Contingencies and Crisis Management, vol. 26, no. 1, pp. 41-57, 2018.

[29] M. Taylor, G. Wells, G. Howell, and B. Raphael, "The role of social media as psychological first aid as a support to community resilience building," Australian Journal of Emergency Management, The, vol. 27, no. 1, pp. 20-26, 2012. 
[30] C. Reuter, T. Ludwig, M.-A. Kaufhold, and T. Spielhofer, "Emergency services attitudes towards social media: A quantitative and qualitative survey across europe," International Journal of Human-Computer Studies, vol. 95, pp. 96-111, 2016.

[31] M. Irons and D. Paton, "Social media and emergent groups: The impact of high functionality on community resilience," Disaster Resilience: An Integrated Approach, 2nd ed.; Paton, D., Johnston, DM, Eds, pp. 194-211, 2017.

[32] W. T. Coombs, "Protecting organization reputations during a crisis: The development and application of situational crisis communication theory," Corporate reputation review, vol. 10, no. 3, pp. 163-176, 2007.

[33] S. P. Goggins, C. Mascaro, and G. Valetto, "Group informatics: A methodological approach and ontology for sociotechnical group research," Journal of the American Society for Information Science and Technology, vol. 64, no. 3, pp. 516-539, 2013.

[34] C. A. Wynd, B. Schmidt, and M. A. Schaefer, "Two quantitative approaches for estimating content validity," Western journal of nursing research, vol. 25, no. 5, pp. 508-518, 2003.

[35] D. F. Polit and C. T. Beck, "The content validity index: are you sure you know what's being reported? critique and recommendations," Research in nursing \& health, vol. 29, no. 5, pp. 489-497, 2006.

[36] M. S. B. Yusoff, "Abc of content validation and content validity index calculation." Education in Medicine Journal, vol. 11, no. 2, 2019.

[37] D. F. Polit, C. T. Beck, and S. V. Owen, "Is the cvi an acceptable indicator of content validity? appraisal and recommendations," Research in nursing \& health, vol. 30, no. 4, pp. 459-467, 2007.

[38] A. F. Hayes, Introduction to mediation, moderation, and conditional process analysis: $A$ regression-based approach. Guilford publications, 2017.

[39] P. E. Shrout and N. Bolger, "Mediation in experimental and nonexperimental studies: new procedures and recommendations." Psychological methods, vol. 7, no. 4, p. 422, 2002.

[40] I. Hellsten, S. Jacobs, and A. Wonneberger, "Active and passive stakeholders in issue arenas: A communication network approach to the bird flu debate on twitter," Public Relations Review, vol. 45, no. 1, pp. 35-48, 2019.

[41] T. G. van der Meer, P. Verhoeven, H. W. Beentjes, and R. Vliegenthart, "Communication in times of crisis: The stakeholder relationship under pressure," Public Relations Review, vol. 43, no. 2, pp. 426-440, 2017.

[42] C. Du Plessis, "Social media crisis communication: Enhancing a discourse of renewal through dialogic content," Public relations review, vol. 44, no. 5, pp. 829-838, 2018.

[43] M. L. Tan, R. Prasanna, K. Stock, E. Hudson-Doyle, G. Leonard, and D. Johnston, "Mobile applications in crisis informatics literature: A systematic review," International journal of disaster risk reduction, vol. 24, pp. 297-311, 2017.

[44] A. Schleicher, "The impact of covid-19 on education insights from education at a glance 2020," Retrieved from oecd. org website: https://www. oecd. org/education/the-impact-ofcovid-19-on-education-insights-education-at-a-glance-2020. pdf, 2020. 